\title{
LA CENSURA PREVIA Y EL ESTADO REGULADOR: PROPUESTAS PARA UNA CONFIGURACIÓN
}

\author{
PRIOR RESTRAINT AND THE REGULATORY STATE: CONCEPTUAL \\ PROPOSALS
}

\section{COMENTARIO DE LA SENTENCIA DEL TRIBUNAL CONSTITUCIONAL DE FECHA 9 DE ENERO DE 2013 (ROL 2358-12)}

\section{Sebastián Zárate Rojas*}

\begin{abstract}
RESUMEN: La sentencia del Tribunal Constitucional en relación al proyecto de ley sobre Televisión Digital Terrestre reviste un especial interés sobre la libertad de expresión en Chile. En este se impugnó una norma que prohibía la medición de audiencia en tiempo real, también denominado people meter online. El TC acogió el requerimiento interpuesto por un grupo de diputados, que representaban la cuarta parte de la Cámara. En el presente comentario se analiza la forma en que se puede vincular la censura previa con la intervención legislativa que pueda afectar al ejercicio de las libertades informativas. Se propone la adopción del principio de neutralidad de contenido como test específico para analizar las posibles vulneraciones a esta libertad.
\end{abstract}

Palabras clave: Libertad de expresión, televisión, libertad de prensa, neutralidad de contenido.

ABSTRACT: The recent decision of the Constitutional Court applied to the Digital Terrestrial Television bill has a special interest for the purposes of freedom of speech in Chile. In this case, a provision of the bill imposed a prohibition on the real-time audience measurement system for broadcasting, known as 'people meter online'. The Court upheld in favour of the applicants, a group of MPs representing a quarter of the House. This comment aims to analyze the relationship between prior restraint with the legislative intervention likely to affect the exercise of free speech. It proposes the adoption of principle of content neutrality as a specific test for this fundamental right.

Key words: Freedom of speech, broadcasting, freedom of the press, content neutrality.

\section{INTRODUCCIÓN}

La sentencia del Tribunal Constitucional en relación al proyecto de ley sobre Televisión Digital Terrestre reviste un especial interés sobre la libertad de expresión en Chile. En este se impugnó una norma que prohibía la medición de audiencia en tiempo real, también denominado people meter online. El TC acogió el requerimiento interpuesto por un grupo de diputados, que representaban la cuarta parte de la Cámara.

\footnotetext{
Profesor de Derecho Constitucional en la Pontificia Universidad Católica de Chile. Doctor en Derecho (PhD.) por la Universidad de Bristol (Inglaterra); Candidato a Doctor por la Universidad Complutense de Madrid (España) y Diploma de Estudios Avanzados (DEA) de la Universidad de Salamanca (España); Abogado y Magíster en Derecho Constitucional por la Pontificia Universidad Católica de Chile.
} 
La sentencia resulta importante desde el punto de vista del derecho constitucional y del derecho de la comunicación, por varias razones. En primer término, porque el texto de los votos de mayoría, minoría y el voto concurrente a la mayoría contienen una doctrina contundente en relación al concepto sobre la libertad económica, igualdad, autonomía de los cuerpos intermedios, y la libertad de expresión.

En segundo lugar, el fallo desarrolla algunos aspectos vinculados al concepto constitucional del "correcto funcionamiento" de la televisión y el régimen regulatorio que la Carta Fundamental establece para este medio de comunicación social. En tercer lugar, reitera la doctrina anterior del TC en cuanto a que las restricciones y limitaciones deben estar previamente autorizadas por el texto constitucional. Como cuarto punto, y que se destacará en el presente comentario, es el debate sobre la definición de la censura previa en un contexto regulatorio como el planteado en este caso.

Tanto la parte requirente, como quienes se opusieron a la declaración plantearon la existencia de censura previa: aquella, por cuanto se trataría de una vía indirecta de control estatal que afectaría la información utilizada por los canales de televisión; esta, porque se trataría del propio sistema de medición de audiencia el que estaría imponiendo contenidos a los canales.

El TC rechazó ambos planteamientos, no considerando que la norma impugnada contenía un vicio de constitucionalidad en cuanto a la libertad de expresión. El voto concurrente de la mayoría aceptó la existencia de censura previa. Por su parte, el voto de minoría, menciona a la censura previa como uno de los elementos en los que la mencionada prohibición estaría precisamente evitando: la censura del indicador en perjuicio de los medios de comunicación.

Lo cierto es que independiente de las tesis a que se pueda adherir, el TC deja abierto para el debate académico aquellos elementos que podrían configurar una censura previa en la regulación de los medios de comunicación. Sabemos que el debate más importante que tuvo nuestro país fue a propósito del cuestionamiento que la Corte Interamericana efectuó de la norma original contenida en el artículo 19 Nº12, que permitía la censura previa únicamente para el caso de la exhibición cinematográfica. Ahora, el tema queda planteado en un contexto de una Constitución reformada en este punto, y en un régimen democrático como el chileno, en el que no se denuncia abuso o arbitrariedad por parte de la autoridad, sino un exceso regulatorio del legislador.

En tal escenario, el presente comentario intentará buscar algunos elementos que definan a la censura previa.

\section{ARGUMENTOS DEL REQUERIMIENTO Y DECISIÓN DEL TRIBUNAL CONSTITUCIONAL}

\subsection{LA NORMA IMPUGNADA}

El requerimiento, interpuesto por 30 diputados tuvo por objeto la declaración preventiva de la norma contenida en el proyecto de ley de Televisión Digital Terrestre, de conformidad con lo dispuesto por el artículo 93 N³ de la Constitución.

La norma establecía lo siguiente: 
"A los prestadores de servicios de radiodifusión televisiva de libre recepción y de servicios limitados de televisión, se les prohíbe el uso de sistemas de medición de audiencia en línea.

Se entenderá por sistema de medición de audiencia en línea, todo aquel sistema que entregue información de audiencia antes de seis horas de haberse terminado la emisión del programa sujeto a medición. La contravención a esta norma impondrá las sanciones contempladas en el artículo 33 de esta ley" ${ }^{1}$.

El texto de la indicación, que había sido rechazado dos veces con anterioridad ${ }^{2}$, fue renovado en la Cámara de Diputados en la votación en sala del proyecto, a propuesta de 37 diputados ${ }^{3}$.

Su fundamento principal consistió en establecer la medida de forma de mejorar la calidad de los contenidos televisivos. Como señaló la H. diputada Saa el día de la votación de la norma:

"[E]l people meter online significa:

1. Manipulación de programas que alteran su contenido original en segmentos tales como matinales, noticiarios y farándula nocturna.

2. Genera un grado de tensión permanente en conductores, actores y participantes en general que son parte del respectivo programa.

3. Desde el punto de vista de género, las principales afectadas son las mujeres, a quienes se sobredimensiona y exacerba como objetos sexuales, mucho más que a los hombres, entregando un contenido eminentemente sexista.

Hay que hacer notar que en los programas en que ha disminuido el sexismo se ha debido a una baja del rating y no por conciencia de género.

4. Desde el punto de vista económico, el aumento artificioso del rating implica también un mayor cobro a las empresas auspiciadoras, dado que sin artificios ni resqui-

1 Corresponde al número 13 del artículo $1^{\circ}$ del proyecto (contenido en el segundo informe de la Comisión de Hacienda del Senado, de fecha 27 de diciembre de 2012, que ratifica el texto aprobado por las segundo informe de las Comisiones de Educación, Cultura, Ciencia y Tecnología, y de Transportes y Telecomunicaciones, unidas, de fecha 12 de octubre de 2012), que buscaba sustituir el artículo 13 de la Ley 18.838, Orgánica Constitucional del Consejo Nacional de Televisión.

2 La indicación había sido originalmente propuesta por los H. diputados Saa, Valcarce, Escobar, González, Sule y Valenzuela, y rechazada por cinco votos a favor y ninguno en contra, según consta en el Informe de la Comisión de Ciencia y Tecnología de la Cámara de Diputados, de fecha 21 de enero de 2010.

Luego, fue repuesta por los diputados Accorsi, Ascencio, Alinco, Andrade, Bertolino, Becker, Ceroni, De Urresti, Díaz, Espinosa, Fidel Espinoza, Farías, González, Goic, Hugo Gutiérrez, Jiménez, Hales, Harboe, Isasi, Jaramillo, Jarpa, Molina, Muñoz, Núñez, , Ojeda, Ortiz, Pascal, Pacheco, Robles, Rivas, Rubilar, Saa, Schilling, Sepúlveda, Silver, Tarud, Torres, Vallespín, Velásquez y Vidal. En esta segunda oportunidad, la Comisión de Cultura y las Artes la rechazó por unanimidad (9 votos contra 0 ), según consta en el Informe de la Comisión de Cultura y las Artes de la Cámara de Diputados (Informe complementario al primer informe de la Ciencias y Tecnología), de fecha 10 de marzo de 2011.

3 La indicación renovada fue aprobada en la Cámara de Diputados por 53 votos a favor, 49 en contra y 3 abstenciones, con fecha 6 de abril de 2011. 
cios, el minuto de publicidad sería un tanto menor, pero sería el que efectivamente correspondería pagar.

5. Todo lo anterior genera como consecuencia un empeoramiento de la calidad de la programación, lo que perjudica a los y a las televidentes de dichos programas.

En conclusión, en la práctica, la medición en línea va en directo perjuicio de consumidores y de auspiciadores.

Creo que si analizamos en profundidad y con interés el tema, y si a los colegas les importa realmente que la televisión llegue de buena manera a los telespectadores, los diputados y las diputadas tenemos una oportunidad al momento de votar. En consecuencia, de nuestra votación depende que siga existiendo este sistema, que es absolutamente perjudicial para la televisión chilena.

He dicho" ${ }^{4}$.

En resumen, la norma tiene un carácter prohibitivo sobre una determinada metodología de medición de audiencia, aquella que se vincula a la medición en tiempo real. Según la información que se tuvo en la tramitación legislativa, en la actualidad se utiliza el sistema de medición en tiempo real en forma conjunta con la medición overnight (es decir, aquella en que se se obtiene la información en forma diferida). Asimismo, se dejó constancia de que dos canales de televisión interrumpieron la utilización del mecanismo cuestionado en el proyecto de ley, durante los años 2005-2008 (en el caso de Canal 13) y 2006-2009 (TVN), sin que se haya observado "ninguna diferencia en la calidad de los contenidos durante los períodos señalados" 5 .

\subsection{Argumentos de las partes}

Los diputados requirentes, impugnaron la norma recién transcrita sobre la base de las siguientes argumentos:

1. Existencia de censura previa, dado que la norma impedía a los canales de televisión la "utilización de una herramienta de información apta para identificar, organizar y seleccionar los contenidos que desean emitir"6.

2. Prohibición absoluta del desarrollo de una actividad económica lícita, que consiste en la medición de audiencias en tiempo real, en lugar de una regulación.

Por su parte, los demás intervinientes indicaron lo siguiente:

3. La medida vulnera el principio de proporcionalidad (Presidente de la República). 4. La norma afecta la adecuada autonomía de los cuerpos intermedios (Federación de Medios de Comunicación A.G., actuando como parte interesada) ${ }^{7}$.

4 Cámara de Diputados (2011), 47-48.

5 Exposición del señor Patricio Moyano, Gerente General de Time Ibope.

6 Escrito del requerimiento, fs. 2.

7 Debo señalar que me correspondió participar en la presentación de la posición de la Federación de Medios 
5. El legislador carece de competencia constitucional para limitar derechos en esta materia (Federación de Medios de Comunicación A.G.).

Por su parte, los asesores parlamentarios de la H. diputada Saa estimaron que el TC debía rechazar el requerimiento, además de las razones indicadas anteriormente, por:

1. Constituir el sistema de medición de audiencias en tiempo real un mecanismo de censura previa, ya que la medición tiene el efecto de determinar "en contenido supuestamente 'libremente' elegido, pero que en los hechos está supeditado a los contenidos de gusto masivo" 8 .

2. La existencia de autocensura de los canales de televisión, entorpeciendo el derecho a recibir información que tienen los ciudadanos.

\subsection{Decisión del Tribunal Constitucional}

El Tribunal Constitucional, en fallo dividido, acogió el requerimiento, declarando la inconstitucionalidad de la norma impugnada, lo que de conformidad a lo dispuesto por el artículo 93 inciso $4^{\circ}$ tiene el efecto de declarar que la norma no puede convertirse en ley.

\subsection{Argumentos POR LoS QUe SE ACOGIÓ El ReQUerimiento}

\section{a) Falta de aptitud de la medida:}

El TC comienza su sentencia efectuando un análisis de la idoneidad de la prohibición para cumplir con la principal finalidad de la medida. Es interesante el análisis previo efectuado, que busca encontrar en la lógica general una explicación racional de la norma, para luego comenzar a expresar las razones constitucionales que lo llevarían luego a declarar la contrariedad con la Norma Fundamental. Ello se ilustra en parte del considerando $9^{\circ}$ :

“(...) Tal deducción es, desde luego, errónea, por serlo también sus premisas. Nada autoriza, salvo si nos situamos en un enfoque puramente elitista, a anticipar que los usuarios seleccionados al azar para ser medidos en sus predilecciones televisivas, van a inclinarse casi fatalmente por la trivialidad y la ramplonería. Ni menos que los directivos de los canales de televisión optarán, también inexcusablemente, por intervenir la programación para preferir o favorecer precisamente los anti-valores que la ley del ramo repudia, en la forma anteriormente descrita, comprometiendo de paso su responsabilidad eventual, en los términos del artículo 33 de la misma preceptiva.

( ) El planteamiento que subyace a la prohibición pretendida implementar a través de la prescripción sujeta al presente control de constitucionalidad, por lo tanto, ado-

de Comunicación. Entendiendo este artículo como un espacio de debate académico, cualquier juicio emitido en este comentario no representa la posición de esta entidad, sino únicamente personales en mi calidad de académico.

8 Escrito presentado por la H. diputada Saa, a fs. 172. 
lece de un vicio de lógica estructural, que lo invalida, transformándolo en una auténtica petición de principios.

La consecuencia de este vicio lógico será estudiada al verificar la constitucionalidad de los preceptos en que podría tener injerencia;"

b) Falta de competencia del legislador para limitar derechos en esta materia: la distinción entre regulación y limitación

Otro argumento consistió en los límites que dispone el legislador en materia de derechos fundamentales, y particularmente en lo referido a la libertades de expresión y económica. En este sentido, la decisión del TC es clara:

"Que, por lo demás, el legislador en esta materia tiene solo una potestad regulatoria, en virtud de la cual puede fijar las condiciones de ejercicio de esa libertad, pero no limitarla, suprimirla o afectarla en su núcleo esencial, mediante una prohibición de utilización de una determinada tecnología de investigación y medición de la teleaudiencia. Tal es efectivamente el criterio sustentado por este Tribunal Constitucional en sentencia Rol No 280, de 20 de octubre de 1988, considerando $29^{\circ}$, donde se enfatizó que la ley solo puede limitar derechos en los casos que la Constitución lo autoriza, cual no es el caso del artículo $19, \mathrm{~N}^{\circ} 12^{\circ}$, inciso $5^{\circ}$, materia de nuestra reflexión"?.

En este sentido, el TC sin decirlo expresamente, aplica el artículo $19 \mathrm{~N}^{\circ} 26$ (en la parte que ella establece "o que los limiten [los derechos] en los casos en que ella [la Constitución] lo autoriza”), exigiendo la habilitación constitucional previa en materia de limitación de derechos. En efecto, resulta importante destacar para en esta materia la referida norma impone para el sistema chileno una distinción entre la regulación o complemento de una garantía y la limitación de la misma. La diferencia resulta importante por varias razones.

En primer término, si bien el artículo $19 \mathrm{~N}^{\circ} 26$ se inspira en el artículo 53.1 de la Constitución española ${ }^{10}$, que a su vez tiene el origen en la Ley Fundamental para la República Federal de Alemania de $1949^{11}$ (artículo 19 ${ }^{12}$ ), la Constitución chilena no permite que sea únicamente el legislador quien establezca limitaciones a los derechos, sino que previamente tales restricciones se encuentren autorizadas por la Constitución. Esta redacción fue debatida en extenso en las sesiones números 212 y 213 de la Comisión de Estudios

\footnotetext{
9 Ley de Televisión Digital Terrestre (2013), considerando $15^{\circ}$.

10 Artículo 53.1 de la Constitución española:

"Los derechos y libertades reconocidos en el Capitulo II del presente Título vinculan a todos los poderes públicos. Solo por Ley, que en todo caso deberá respetar su contenido esencial, podrá regularse el ejercicio de tales derechos y libertades que se tutelarán de acuerdo con lo previsto en el artículo 161.1.a".

11 Navarro Beltrán (2010) 33.

12 Artículo 19, párrafos 1 y 2 :

(1) Cuando de acuerdo con la presente Ley Fundamental un derecho fundamental pueda ser restringido por ley o en virtud de una ley, ésta debe tener carácter general y no estar limitada al caso individual. Además, la ley debe mencionar el derecho fundamental indicando el articulo correspondiente.

(2) En ningún caso un derecho fundamental podrá ser afectado en su contenido esencial.
} 
para la Nueva Constitución, planteándose la necesidad de exigirle a la Constitución disponer la existencia de limitaciones:

"El señor ORTUZAR (Presidente) lee el artículo siguiente, que expresa: 'Las leyes que interpreten, regulen, complementen o, en los casos que la Constitución autoriza, limiten las garantías que ella asegura, no podrán afectar los derechos en su esencia ni imponer condiciones, tributos o requisitos que impidan su libre ejercicio. Se exceptúan las leyes dictadas en virtud de los estados de emergencia que la propia Constitución consigna'.

Subraya que le interesa dejar bien claro que la frase 'en los casos que la Constitución autoriza' solamente rige respecto de las disposiciones que 'limiten las garantías que ella asegura"”

En la distinción entre regulación y limitación, con la autorización constitucional a la que nos referimos, la Constitución de 1980 se aparta del modelo alemán y español, estableciendo un criterio notoriamente más estricto. Hay que indicar, que la diferencia entre limitación y restricción no fue compartida por todos los comisionados de la CENC, y que en parte a ello se extendió el debate de la norma. Ello, porque como también consta de la doctrina comparada, se entiende que la regulación tiene el efecto de limitar ${ }^{13}$.

La diferencia, como expone Aldunate, radicaría en que en una limitación la Constitución "habilita al legislador para fijar la extensión de la respectiva libertad o derecho" ${ }^{14}$, mientras que una regulación entregaría a la ley un mandato de "indicar modalidades de su ejercicio" 15 .

En efecto, vemos que tanto el tenor literal como sistemático de la norma que se impugnó en la sentencia que comentamos se refiere a la prohibición de un derecho fundamental, que en este caso se trata de la libertad económica de quien desarrolle un estudio de audiencia, y principalmente a nuestro entender, de la libertad editorial que disponen los medios de comunicación. Tal prohibición se configura en este caso como una limitación absoluta de la medición online, que se aparta de la exigencia de contar con autorización constitucional previa.

Por otra parte, tampoco es posible entender que tal prohibición sea parte de las prohibiciones contenidas en el artículo $19 \mathrm{~N}^{\circ} 21$, dado que no hay razón alguna para entender que la medición de audiencia online sea una actividad contraria a la moral, al orden público o a la seguridad nacional.

\section{c) Falta de razonabilidad y proporcionalidad}

El TC continúa analizando, ahora desde la perspectiva del principio de proporcionalidad, argumentando que se trata de una medida que carece de idoneidad para cumplir con la medida propuesta.

13 Peces-Barba (2004).

14 Aldunate Lizana (2008) 247.

15 Aldunate Lizana (2008) 247. 
"Es por ello que la disposición legal cuestionada deviene fundamentalmente desproporcionada y no razonable, toda vez que no existe demostración conocida de alguna adecuación causal entre la prohibición legal -como medio- y el mejoramiento de la calidad de la programación de la televisión -como fin-, en tanto cuanto el instrumento tecnológico como tal es neutro y las decisiones de programación son autónomas de la línea editorial del canal, pero no escapan de las facultades de supervigilancia del Consejo Nacional de Televisión, precisamente encargado constitucionalmente de velar por el correcto funcionamiento de este medio de comunicación, lo que es ciertamente un medio menos gravoso e invasivo y más idóneo para el fin señalado. Existe en esto una relación análoga a la que se da entre el termómetro y la temperatura del paciente, instrumento que ciertamente no es la causa de su enfermedad" ${ }^{16}$.

Entendemos que este razonamiento del Tribunal, incluido en el mismo considerando que el argumento relativo a la competencia, sería estrictamente innecesario, dado que el test debiera aplicarse únicamente para el caso en que el legislador fuera competente. Sin embargo, debe entenderse como un argumento dentro del análisis de constitucionalidad de la medida, que recoge el planteamiento tanto de los requirentes como las observaciones formuladas por el Presidente de la República, y que se pronuncia sobre los vicios de constitucionalidad de la norma.

\section{d) Vulneración de la autonomía de los cuerpos intermedios}

Enseguida, el TC viene a aplicar la protección a la autonomía de los cuerpos intermedios, tal como lo hizo en el caso "Ley de Prensa" (STC 226/1995). Nos referiremos nuevamente sobre este punto al tratar el tema de la "regulaciones neutras", desde la perspectiva del análisis de constitucionalidad de la libertad de expresión.

\section{e) No discriminación arbitraria}

Sin que haya sido incluida por los requirentes ni partes interesadas, se trata de una razón que fue desarrollada en la misma audiencia por el abogado patrocinante, a petición de uno de los ministros.

En efecto, el TC señala que con esta medida se discrimina a los canales de televisión, por cuanto el Estado pretende regular sus sistemas de medición de audiencias, que no se encontraría prohibido en el caso de los otros medios de comunicación. Este argumento también será abordado con posterioridad.

\section{f) Prohibición de una actividad económica lícita}

El TC también afirma compartir "el predicamento del requerimiento en el sentido que la prohibición proyectada importa una supresión de la demanda por un servicio, lo que indudablemente amaga la actividad económica de las empresas proveedoras de ese servicio de medición, sin que se divise -conforme a lo analizado- razón moral, de orden público o de seguridad nacional que amerite una tal prohibición legal”.

16 Ley de Televisión Digital Terrestre (2013), considerando $15^{\circ}$. 


\section{RECHAZO DEL ARGUMENTO DE LA CENSURA PREVIA}

Sin duda, uno de los elementos de mayor divergencia entre el voto mayoritario, el concurrente y el de minoría consiste en la distinta apreciación respecto de la forma en que se ve afectada la libertad de expresión. Como expresó el TC:

"Que, por otra parte, no cabe duda que en la tradición constitucional occidental, la libertad de emitir opinión y la de informar, sin censura previa, se originaron primero en relación a la libertad de prensa y, ulteriormente, fue incluyendo otros medios de comunicación social, a medida que se desarrollaban tecnológicamente como soportes diversos de las opiniones e informaciones en flujo social, dentro del concepto genérico de libertad de expresión, que a su vez alcanza incluso a otras manifestaciones de las personas. De manera que, conceptualmente, no hay inconveniente alguno en aceptar de suyo la pertinencia de la prohibición de censura, en cualquiera de sus modalidades, incluso indirecta, para la televisión. Pero, también conceptualmente, la censura implica una interferencia previa (prior restraint, en el derecho constitucional norteamericano) a la emisión de la información o contenido, en este caso, del programa de televisión; por lo que no puede aceptarse que la prohibición de audímetro sea inconstitucional por este motivo en la especie. En efecto, como fuere, la prohibición incidiría en la medición ulterior de la audiencia del contenido ya transmitido (respuesta a la información o contenido), mas no en la posibilidad previa de hacerlo. De hecho, existen otras técnicas de investigación social que permitirían medir esa audiencia del programa emitido, aun con tal prohibición.

Este capítulo del requerimiento, por consiguiente, no podrá prosperar"17.

Por su parte, la ministra señora Marisol Peña, quien concurrió con su voto a la declaratoria de inconstitucionalidad de la norma, incorporó la siguiente prevención:

“(...) El 'people meter online', en cuanto sistema neutro y, por ende, objetivo, describe tendencias y, bajo esa óptica, puede ser considerado como parte de las fuentes de información cuya libertad de acceso, por parte de los medios televisivos, les está garantizada por el artículo $19 \mathrm{~N}^{\circ} 12^{\circ}$, inciso primero, en relación con su derecho a 'operar' tales servicios, asegurada, a su vez, por el inciso quinto del mismo numeral de la Constitución Política.

Así, prohibir o impedir el acceso a una fuente de información, como la que hoy constituye el 'people meter online', configura una censura previa indirecta, prohibida por el artículo $19 \mathrm{~N}^{\circ} 12^{\circ}$, inciso primero, de la Carta Fundamental, porque limita, en forma anticipada, la facultad de los servicios de televisión de conocer preferencias, tendencias, gustos u orientaciones del público, cuya consideración tiende a influir

17 Ley de Televisión Digital Terrestre (2013), considerando $12^{\circ}$. 
directamente en la definición de los contenidos de la programación que imparten en virtud de su facultad de 'operar' estaciones de esa naturaleza"18.

Por último, cabe consignar el razonamiento de quienes concurren con su voto minoritario, en relación a su rechazo a la idea de que se vulnera la libre expresión:

"Que, asimismo, la medida puede perfectamente enmarcarse, por una parte, en la dimensión social de la libertad de opinión y de información. Es decir, en el derecho a recibir información. En la medida que las decisiones de programación se orienten únicamente por el rating, se compromete seriamente esta dimensión de la libertad de opinión e información, pues se produce una verdadera autocensura del medio. Por la otra, se enmarca dentro del criterio del correcto funcionamiento de la televisión. Dado que la televisión opera con concesiones otorgadas por la autoridad, utilizando un espectro radioeléctrico limitado, no se puede enfrentar la disputa entre la rentabilidad, que emana de la necesidad de autofinanciamiento, y la libertad de opinión y de información, sacrificando esta última.(...)"19.

Como se podrá apreciar, el TC no logró llegar a un acuerdo sobre si la medida constituía una forma de censura previa.

Como vemos, el voto de mayoría mantiene un criterio temporal de la regulación. Es decir, la prohibición de la medición es posterior a la emisión del mensaje, y por tanto no puede constituir en censura previa. En segundo lugar, bajo este criterio la censura previa estaría definida únicamente sobre el mensaje transmitido, no en los medios que anteceden a la transmisión. En tercer lugar, el contenido informativo es solo el que proviene del medio de comunicación, no de la información emanada por la empresa encargada de la medición de audiencia.

Por su parte, el voto concurrente alude precisamente al funcionamiento del medio de comunicación, que busca y selecciona los mensajes, en este caso teniendo el consideración -entre otros elementos- las preferencias del público.

Por último, el voto de minoría justifica una regulación previa sobre la base de una dimensión social de la información, en el derecho a ser informado. Introducen asimismo el concepto de autocensura de los medios, como un elemento atentatorio al derecho a recibir información.

Vemos en definitiva en la sentencia, tres criterios divergentes respecto de lo que constituye censura previa. Naturalmente que no es la finalidad del presente comentario indicar cuál es la "interpretación constitucionalmente adecuada", dado que ello solo implicaría ignorar el carácter abierto de la interpretación constitucional. Tampoco desconocer que la interpretación del TC es la contenida en el voto mayoritario. La misión de este comentario más bien consistirá en una propuesta de solución a la definición de la censura previa,

18 Ley de Televisión Digital Terrestre (2013), considerando $10^{\circ}$ del voto de prevención de la Ministra Sra. Peña.

19 Ley de Televisión Digital Terrestre (2013), considerando $41^{\circ}$ del voto de minoría de los Ministros señores Fernández, Carmona, Viera-Gallo y García. 
al menos en los términos que se plantearon en este caso, y que puede servir para dilucidar otros casos en los que se plantee una regulación que afecte al flujo informativo.

\section{CONSIDERACIONES SOBRE LA CENSURA PREVIA EN EL CASO PLANTEADO}

Naturalmente que sería fácil definir a la censura previa como una abierta y grosera forma de control, análisis y edición previo de los contenidos de los medios. Afortunadamente para nuestro derecho este no ha sido el caso, y el TC se enfrenta a un complejo escenario que más bien plantea la existencia de los elementos que justifican la existencia de tal censura.

\subsection{LIBERTADES DE EXPRESIÓN COARTADAS}

Una primera consideración que habría que hacer sobre el caso, es que en él realmente concurren dos discursos susceptibles de protección bajo la libertad de expresión: por una parte la de los canales de televisión, tal como se planteó; y por otra, la de las empresas que se dedican a la medición del rating online. Partamos por estas últimas, dado que no fue una materia que haya aparecido en el debate ante el TC ni tampoco en la sentencia.

\subsection{Dos Discursos: EL DE LAS EMPRESAS DE MEDICIÓN DE AUDIENCIAS Y EL DE LOS CANALES DE TELEVISIÓN \\ ¿Por qué puede considerarse a la medición de audiencias como un discurso? La me-} dición de audiencia no es otra cosa que un análisis de la realidad efectuado bajo ciertos criterios metodológicos previamente definidos y que tienen como resultado un diagnóstico del consumo de los contenidos televisivos. Como tal, constituye una actividad económica amparada bajo el artículo 19 N²1 de la Constitución, cuya finalidad es la obtención de información es ofrecer información a los canales de televisión. En consecuencia, la prohibición de una modalidad de medición de audiencia es una limitación en la existencia de información, generando un menoscabo en la libre expresión.

En Estados Unidos este debate dio en dos casos en materia de medición de audiencia, uno respecto de aquella desarrollada en tiempo real en televisión, mientras que el otro se produjo con respecto al portable people meter, un dispositivo que permite la recepción de señales de radio, de modo de ver en cada minuto las preferencias de los auditores. En ambos casos, tanto para la televisión como en radio, se interpusieron acciones en contra de las empresas prestadoras de los servicios (Nielsen ${ }^{20}$ y Arbitron ${ }^{21}$ ), por cuanto sectores consideraron que la medición tenía falencias metodológicas que producían la subrepresentación de ciertos sectores sociales. Así, en el caso de Nielsen fue la cadena Univisión la que reclamó por el mencionado supuesto defecto en la metodología empleada.

20 Univision Communications, Inc. v. Nielsen Media Research, Inc. (2004).

21 Arbitron Inc. v. Cuomo (2008). 
Las empresas en ambos casos argumentaron que la regulación de la medición era atentatoria contra la libertad de expresión. Si bien en el caso de Arbitron se llegó a un acuerdo judicial, la Corte de California acogió el argumento en el caso Nielsen.

En ambas acciones judiciales el gran debate consistió en si la información que proporcionada por los sistemas de medición podían ser clasificados como un discurso comercial o no comercial. La diferencia, bajo el sistema de Estados Unidos consiste en que el discurso comercial recibe una menor protección constitucional bajo la primera enmienda ${ }^{22}$. De esta manera, si se estimaba como discurso comercial, cualquier interferencia judicial o legislativa en la regulación del mecanismo no sería en principio objetable. En caso contrario, si formaba parte de la categoría de discurso no comercial, se planteaba como una presunción de inconstitucionalidad. La Corte de California finalmente optó por esta última solución:

"Aunque los avisadores y compradores dependen de la información arrojada por las mediciones, el sistema de medición de audiencias en sí mismo no implica una transacción comercial. Por lo tanto, el discurso puede tener plena protección bajo la Primera Enmienda"23.

En efecto, compartimos la visión conforme a la cual la información de la medición es una mezcla de información objetiva y opinión ${ }^{24}$. Lo es porque pretende ser un reflejo de una realidad (hechos objetivos), pero sujeta a un tratamiento metodológico de medición que busca expresar una idea sobre cómo una entidad interpreta las preferencias de una determinada audiencia.

¿Qué utilidad tiene el razonamiento recién planteado para el caso que comentamos? Hay una aplicación muy directa. Una de las razones por las que el TC rechaza la afectación de la censura previa consiste en un análisis temporal de la intervención del Estado, sosteniendo que la prohibición no es previa sino posterior a la emisión del mensaje ${ }^{25}$. Lo anterior es cierto, pero solo para el mensaje de la empresa que efectúa la medición. Para el medio de comunicación es previa, dado que es la información de la audiencia la que le permite a cada canal tomar sus decisiones editoriales y comerciales. Dicho de otro modo, la medición de audiencia en tiempo real no es un mecanismo de evaluación posterior de la programación, sino de toma de decisiones inmediatamente posteriores a la recepción de la información. Así, el criterio de temporalidad es parcialmente cierto, pero solo para las empresas de medición. Para los canales de televisión, en cambio, la prohibición de la ausencia de tal información incide en las decisiones posteriores que los medios de comunicación adoptan para definir su programación, por tanto es un mecanismo de afectación previo.

\footnotetext{
22 Aunque no todos están conformes con esta categoría. Véase Reed (1996).

23 Univision Communications, Inc. v. Nielsen Media Research, Inc. (2004) (traducción propia del inglés).

24 Napoli (2010) pp. 376-377.

25 Como razonó el TC, "la prohibición incidiría en la medición ulterior de la audiencia del contenido ya transmitido (respuesta a la información o contenido), mas no en la posibilidad previa de hacerlo".
} 


\section{APLICACIÓN DEL PRINCIPIO DE NEUTRALIDAD DEL CONTENIDO: IGUALDAD Y AUTONOMÍA DE LOS CUERPOS INTERMEDIOS}

Otro argumento que resulta interesante de tener en cuenta en materia de regulación de los medios de comunicación, y de los mensajes en términos generales dice relación con la neutralidad de contenido. En breves palabras, se trata de un criterio conforme al cual no le es legítimo al Estado establecer regulaciones relacionadas con la libertad de expresión que se funden en contenidos determinados, privilegiando un tipo de mensaje y prohibiendo otro. Lo que se prohíbe es en general que el Estado manipule el libre flujo de las ideas por medio del control de los mensajes que pueden circular ${ }^{26}$.

En tal sentido, el criterio que ha adoptado la Corte Suprema de Estados Unidos desde hace más de cuarenta años ha consistido en tratar con cierta sospecha la constitucionalidad (presumiendo la inconstitucionalidad) de cualquier norma que privilegie un contenido determinado, solo resultando justificada tal distinción bajo ciertos requisitos muy excepcionales.

De este modo, una regulación que sea neutra de contenido será aquella que no imponga restricciones distintas según el mensaje. Por ejemplo, una regulación que restrinja los ruidos molestos en un sector residencial en determinado horario, se aplicará a cualquier persona o grupo que pretenda efectuar una actividad de expresión musical, artística, política, de marketing o religiosa, sin pretender tampoco desfavorecer a un grupo determinado. En tal caso, no importa el contenido del mensaje.

Por el contrario, la regulación basada en el contenido solo se aplica a ciertas hipótesis, excluyendo la expresión de algunas ideas.

Hay varios argumentos que justifican la bondad de este criterio. De una parte, implica un reconocimiento de la primacía de la persona sobre el Estado, de la autonomía para adoptar las propias decisiones sin que sea el Estado el que privilegie ciertos contenidos, excluyendo otros. En segundo término, hay un argumento vinculado al principio de igualdad.

Un caso demostrativo de esta realidad se dio en Estados Unidos en 1972 (Police Department of the City of Chicago v. Mosley ${ }^{27}$ ), cuando la Corte Suprema declaró inconstitucional una norma que prohibía cualquier manifestación pacífica frente a establecimientos educacionales, salvo en caso de huelga de sus profesores. Aplicando la norma, se sancionó a una persona que reclamaba pacíficamente por la discriminación racial de los establecimientos educacionales. La Corte Suprema de Estados Unidos estimó que la norma estaba basada en el contenido, declarando que "el gobierno no tenía la facultad de restringir la libertad de expresión en razón del mensaje, ideas, asunto determinado, o su contenido" 28 . De este modo, concluyó que la medida era discriminatoria para cualquier otra persona que quisiera expresarse por otros motivos.

Este argumento fue precisamente uno de los utilizados por el TC en el caso que comentamos. En primer lugar, porque la prohibición solamente es aplicable a una cierta categoría de entidades: los canales de televisión. Pero también se puede plantear un problema

26 Chemerinsky (2000) p. 64.

27 Police Dept. of City of Chicago v. Mosley (1972).

28 Police Dept. of City of Chicago v. Mosley (1972). 
de igualdad en el mensaje que se pretende privilegiar y aquel que se quiere desincentivar. En efecto, al asimilar la utilización de una determinada metodología de medición de audiencia con determinada clase de programación, más vinculada al entretenimiento que a la cultura, como se expuso como fundamento de la prohibición, se privilegia un contenido por sobre otro.

La Corte Suprema de Estados Unidos ha impuesto una serie de criterios (tests) para evaluar la constitucionalidad de una regulación, y definir si se trata de aquellas bajo la categoría de neutrales en contenido o no. Así, ha aplicado tests más estrictos que otros sobre la materia:

a) Estará basada en el contenido (y por tanto inconstitucional) si la regulación "depende del efecto comunicativo que tenga la conducta": Texas v. Johnson ${ }^{29}$.

b) Estará basada en el contenido (y por tanto inconstitucional) si la regulación está motivada por los efectos de la comunicación más que con los efectos secundarios que esta tenga: City of Renton v. Playtime Theatres, Inc. ${ }^{30}$, aunque estos sean benignos ${ }^{31}$.

c) Estará basada en el contenido (y por tanto inconstitucional) si la regulación está fundada en la discrepancia respecto del contenido: Hill v. Colorado ${ }^{32}$.

d) Por último, en $2011^{33}$ la Corte entregó un test que parece aún más aplicable al caso revisado por el TC. Declaró la inconstitucionalidad de una norma, por considerarla basada en contenido, por cuanto restringía el acceso y uso de información entre farmacias hacia laboratorios con fines de adecuar la información comercial a los pacientes y médicos.

Vemos que en estos cuatro criterios citados, la Corte Suprema de Estados Unidos tuvo que determinar si una regulación en materia de información era reprochable bajo la Constitución. Estimamos que los referidos tests podrían ser fácilmente aplicables en este caso, tanto en la regulación de información de las empresas que desarrollan los estudios de audiencias, como por los canales de televisión. No se trató solamente de un procedimiento, menos de un cuestionamiento a la metodología de los estudios, sino en los efectos que podrían tener estos en la información entregada por los canales. Ello indica que la regulación precisamente busca modificar la forma en que los medios de comunicación entregan su información, la selección de los medios, las decisiones editoriales y comerciales, y el resultado materializado en un determinado contenido de la televisión y no en el actual, que se intenta modificar.

Pero la neutralidad de contenido, tal como se expuso anteriormente, se explica por la dificultad de justificar la legitimidad del Estado para determinar qué mensajes deben existir en perjuicio de otros. Ciertamente que las mayorías parlamentarias no son suficientes para

\footnotetext{
29 Texas v. Johnson (1989).

30 City of Renton v. Playtime Theatres, Inc. (1986).

31 Calvert (1997) 106.

32 Hill v. Colorado (2000).

33 Sorrell v. IMS Health Inc. (2011).
} 
efectuar tal decisión fundamental en la libertad de las personas, ni tampoco promueven la generación de nuevas ideas, cuyos emisores podrían tener un justo temor de manifestarlas por no ser sancionados. Y en este punto, la primacía de la persona y de los cuerpos intermedios sobre el Estado tiene aplicación en el caso que comentamos.

Como explica Heyman, el principio de neutralidad de contenido implica una protección a la autonomía "ya sea porque el Estado desaprueba el hecho que una persona adopte un cierto pensamiento o sentimiento, o que lo pretenda expresar. Aun cuando el Estado restrinja el mensaje por otras razones, dicha restricción infringe la autonomía y autorealización si tales razones no son correctas" 34 .

De esta forma, una regulación de contenido no neutral tiene el defecto de que es el Estado el que selecciona los mensajes que deben privilegiarse, o bien los medios conducentes a la selección de los mensajes por parte de quienes los emiten.

Por ello es que el TC aplicó el criterio de Ley de Prensa ${ }^{35}$. Recordemos que en tal caso se rechazó el criterio en virtud del cual se otorgaba al Estado una función de garante del pluralismo informativo. Como expresó el Tribunal:

"Que, el derecho que se consagra en el proyecto de ley no otorga en ninguna de sus partes una atribución al Estado para exigir que se dé una determinada opinión, noticia o información sobre el acontecer nacional o internacional. Las normas constitucionales deben ser interpretadas en forma armónica y sistemática y si se reconoce a las personas el derecho de opinar y de informar, y por otra parte, se garantiza a los cuerpos intermedios de la comunidad su adecuada autonomía, se desprende con claridad que ninguna norma legal puede obligar a las personas naturales o jurídicas a dar una información u opinión o a inmiscuirse en la autonomía que deben tener los grupos intermedios de la comunidad entre los que se cuentan los medios de comunicación social ${ }^{36 "}$.

En resumen, el criterio de la neutralidad del mensaje tiene aplicación en casos de regulación de discursos, como en el que comentamos, y en general en toda intervención de carácter legal que pretenda regular el flujo de información. No quiere decir que sea imposible hacerlo desde el punto de vista constitucional, y de hecho hay autorización constitucional para ciertas limitaciones al derecho. Asimismo, como expresó el TC, el legislador tiene competencia general para regular, entendiendo que tal ejercicio será el establecimiento de modalidades de ejercicio de la libre expresión (aunque carece de competencia general para limitar derechos, tal como expusimos).

De este modo, cuando el legislador pretende establecer algo más que una mera forma de ejercicio del derecho, privilegiando contenidos sobre otros, altera esa habilitación regulatoria e incorpora elementos de limitación. Asimismo, la forma en que el Estado pre-

\footnotetext{
34 Heyman (2002), 658 (traducción propia del inglés).

35 Ley de Prensa (1995).

36 Considerando $22^{\circ}$.
} 
tende sustituir el mensaje por la autonomía de los individuos o los cuerpos intermedios, genera una vulneración del principio reconocido en el artículo $1^{\circ}$ inc. $3^{\circ}$ de la Constitución.

\section{CONCLUSIONES: EL FUTURO DE LA INTERVENCIÓN LEGISLATIVA EN LAS LIBERTADES INFORMATIVAS}

El fallo del TC, a pesar de haber desestimado las alegaciones sobre el impacto de una limitación de este tipo en la libertad de expresión, sin duda será una decisión de importancia en la regulación de los medios.

Sin embargo, hay ciertas categorías en materia regulatoria que merecen una mayor atención de la doctrina constitucional. La sentencia del TC reúne todos los elementos que permitirían tal interpretación jurídica acerca de las formas en que el Estado regulador impacta en las libertades informativas, y cuáles son constitutivas de censura previa.

En tal sentido, el TC pudo recoger elementos como la discriminación arbitraria, la limitación y no regulación de la libertad económica, y en especial, la limitación exorbitante de la autonomía de los cuerpos intermedios, todo lo cual pretendía estar suprimido por una prohibición.

Nuestra propuesta en este comentario es que tales ingredientes configuran un vicio constitucional de una cada vez más compleja y sofisticada forma de interferir en las libertades informativas, en imponer un contenido y en no reconocer la libre expresión más allá de lo que tradicionalmente de definió en la comunicación social bajo las fórmulas jurídicas. Hablamos de mecanismos contemporáneos de censura previa, propia del denominado "Estado regulador”.

Así, el tratamiento jurisprudencial en Estados Unidos, en especial en lo referido a los tests de neutralidad del contenido de la regulación, podrían ser adoptados en nuestro país. Vemos el gran cambio que significó para el control de constitucionalidad la irrupción de otros tests generales en materia de derechos fundamentales, como la recepción del principio de proporcionalidad. En materia de control de las normas que afecten las libertades informativas, la aplicación del criterio de la neutralidad de contenido podrá dar mayor certeza jurídica, y permitir mayores espacios para que el legislador pueda optar por aquellos mecanismos constitucionalmente idóneos, que cumpliendo el objetivo de política pública que se plantee, pueda respetar los espacios de libertad que la Carta Fundamental reconoce con respecto a esta garantía.

\section{BIBLIOGRAFÍA}

Aldunate Lizana, Eduardo (2008): Derechos Fundamentales (Legal-Publishing, Santiago, 2008).

Calvert, C. (1997): "Free Speech and Content-Neutrality: Inconsistent Applications of an Increasingly Malleable Doctrine”. McGeorge L. Rev., 29, 69.

Cámara de Diputados (2011): Boletín de Sesiones, Sesión $7^{\text {a }}$ ordinaria, en miércoles 23 de marzo de 2011. 
Chemerinsky, E. (2000): "Content neutrality as a central problem of freedom of speech: problems in the Supreme Court's application". Southern California Law Review, 74(1): p 49.

Heyman, S. J. (2002): "Spheres of Autonomy: Reforming the Content Neutrality Doctrine in First Amendment Jurisprudence". William and Mary Bill of Rights Journal, 10(3), 647.

Navarro Beltrán, Enrique (2010): Derechos Fundamentales en Chile: evolución, alcance y protección, en VV.AA. La Ley Fundamental Alemana y la Constitución Política Chilena, (Editorial Jurídica de Chile, Santiago).

Peces-Barba, Gregorio (2004): Lecciones de Derechos Fundamentales (Editorial Dykinson, Madrid).

ReED, O. L. (1996): "Is commercial speech really less valuable than political speech? On replacing values and categories in First Amendment jurisprudence" American Business Law Journal, 34(1), 1.

\section{JURISPRUDENCIA CITADA}

Ley de Prensa (1995): Tribunal Constitucional, Rol 226, 30 de octubre de 1995.

Televisión Digital Terrestre (2013): Tribunal Constitucional, Rol 2358, 9 de enero de 2013

Univision Communications, Inc. v. Nielsen Media Research, Inc. (2004) No. BC318833 2004 WL 3050799 (Cal. Super. Ct. 20047 julio, 2004)

Arbitron Inc. v. Cuomo, No. 08 Civ. 8497, 2008 U.S. Dist. LEXIS 86573 (S.D.N.Y. Oct.

27, 2008)Police Dept. of City of Chicago v. Mosley, 408 U.S. 92 (1972)

Texas v. Johnson, No. 88-155, 491 U.S. 397 (1989)

City of Renton v. Playtime Theatres, Inc., 475 U.S. 41 (1986)

Hill v. Colorado (98-1856) 530 U.S. 703 (2000)

Sorrell v. IMS Health Inc., No. 10-779 (2011)

\section{NORMAS CITADAS}

Constitución Política de la República de Chile

Constitución de Estados Unidos

Constitución Española

Ley Fundamental para la República Federal de Alemania 